\title{
Simultaneous Spectroscopic and Solid-State Electronic Measurement of Single-Walled Carbon Nanotube Devices
}

\author{
Douglas R. Kauffman and Alexander Star*
}

University of Pittsburgh, Department of Chemistry, Pittsburgh PA, 15260, and the National Energy Technology Laboratory, U.S. Department of Energy, Pittsburgh PA, 15236.

\section{Supporting Information}
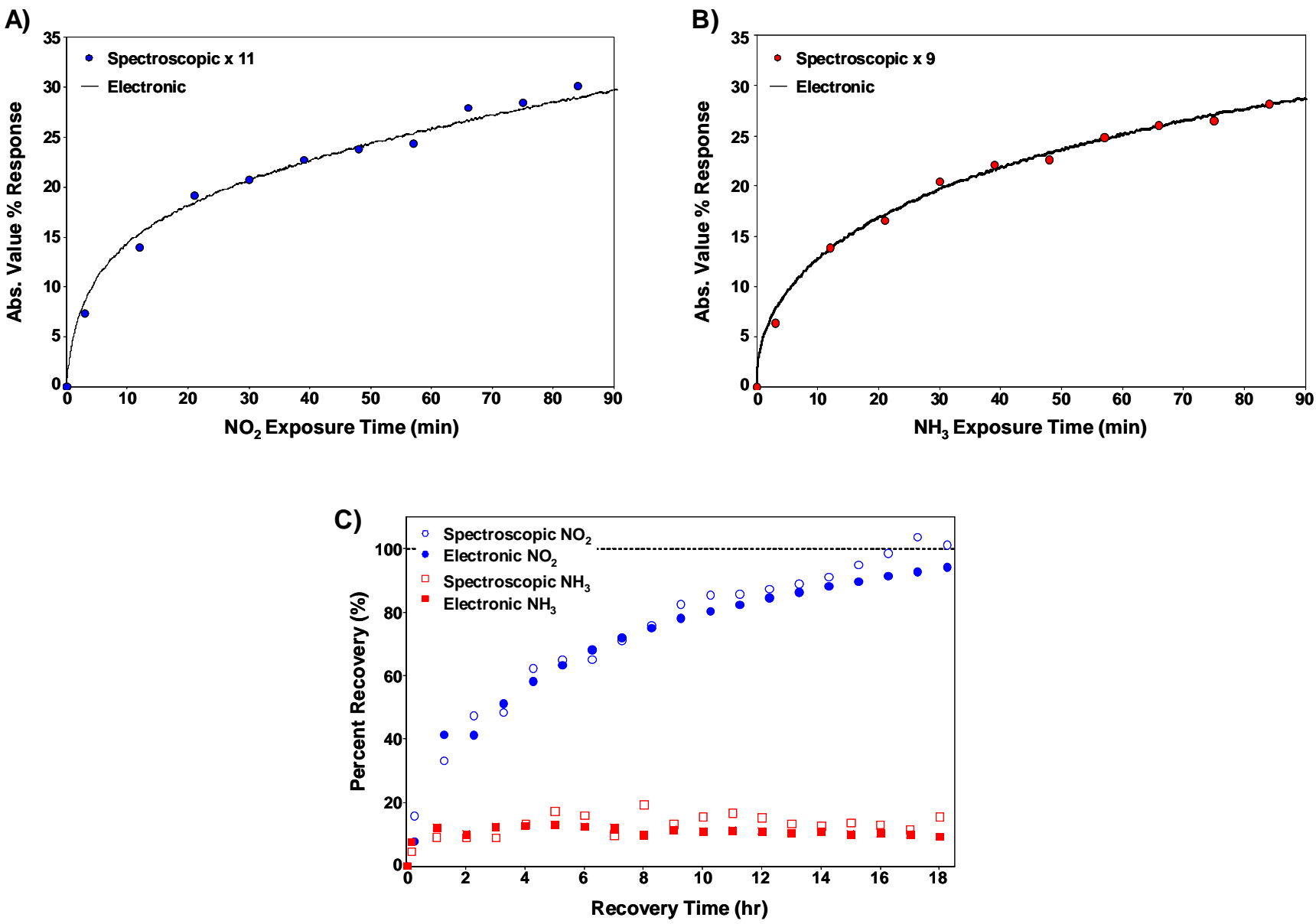

Figure S1. A plot detailing the absolute value of the percent response of a SWNT film device under A) $10 \mathrm{ppm} \mathrm{NO}_{2}$ and B) $100 \mathrm{ppm} \mathrm{NH}_{3}$. Notice for each gas the electronic and spectroscopic response profiles are nearly identical-we contend this is because the combined device response is a result of gas adsorption on the SWNT surface. Although the traces are nearly identical the electronic response is approximately one order of magnitude larger than the spectroscopic response for each gas. C) Independent percent recovery under 300 SCCM flowing $\mathrm{N}_{2}$ after the devices shown in parts A) and B) were exposed to gas. Notice here that recovery from $\mathrm{NO}_{2}$ was approximately $100 \%$, while recovery from $\mathrm{NH}_{3}$ was only $15 \%$. However, the independent electronic and spectroscopic recovery profiles are nearly identical for a particular gas. We contend this indicates the device behavior is dominated by gas adsorption on the SWNT surface. The partial recovery of this type of device from $\mathrm{NH}_{3}$ has previously been reported; see reference \# 10 in the main text. 


\section{"High Density" SWNT Films}

A)

C)
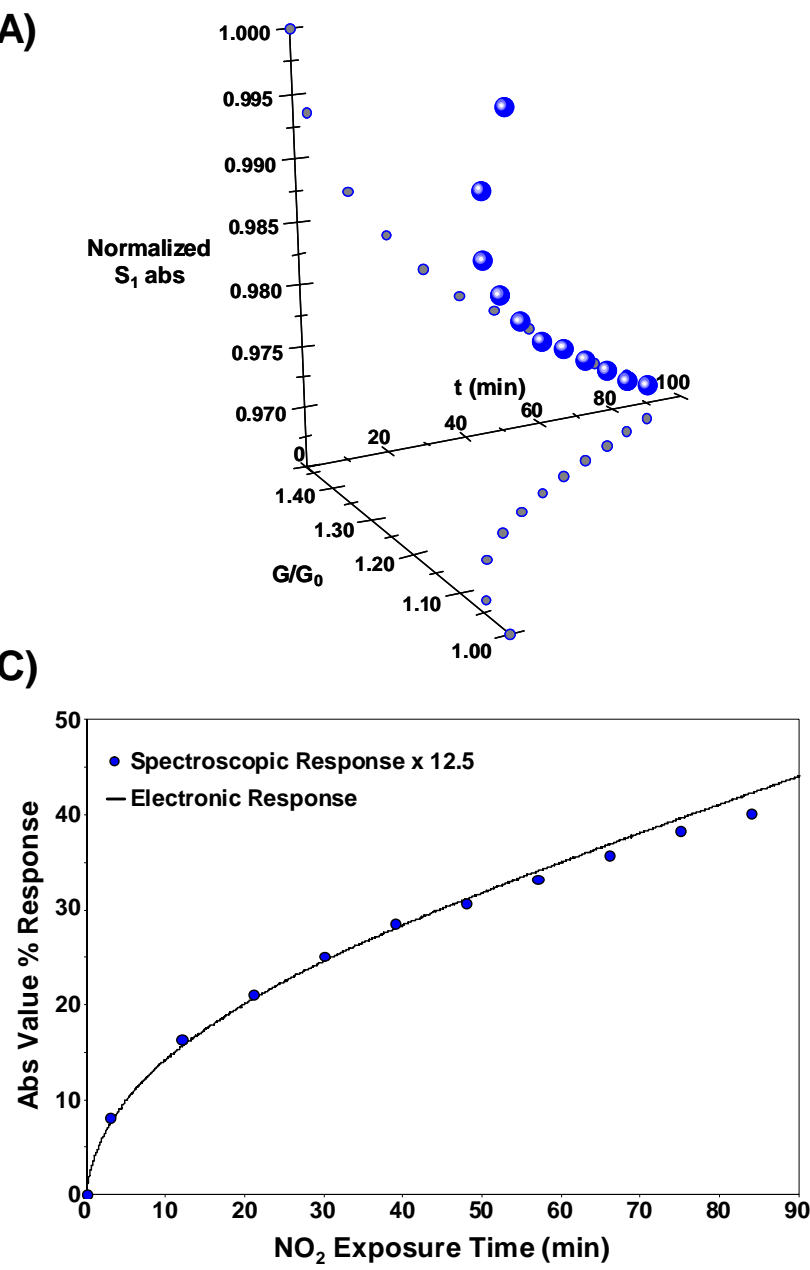

B)

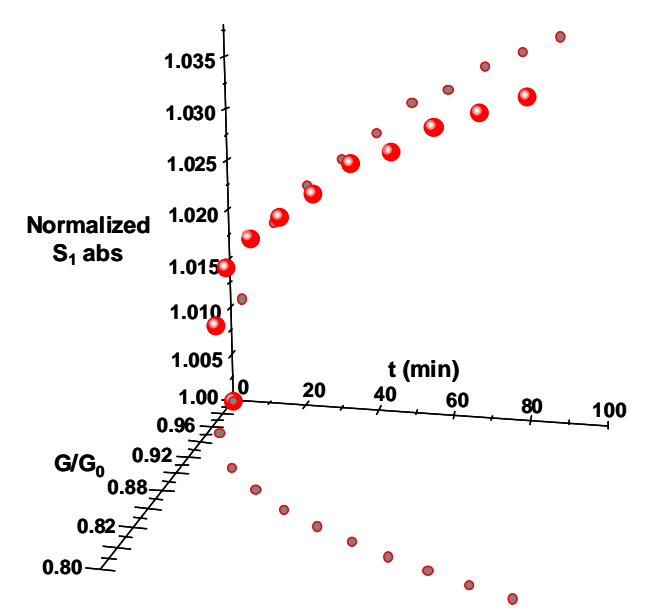

D)

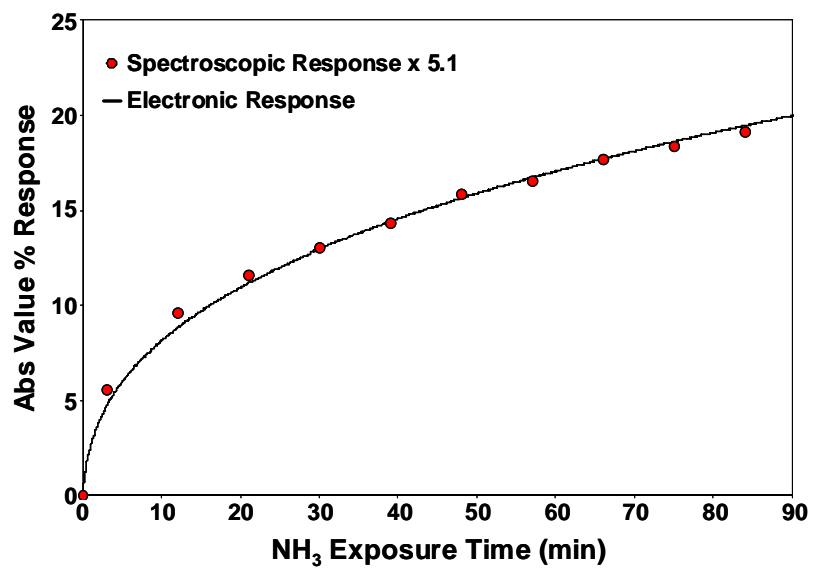

Figure S2. "High density" device output under A) $\mathrm{NO}_{2}$ and B) $\mathrm{NH}_{3}$ gases. Absolute value of the device spectroscopic and electronic response (\% $\Delta \mathrm{G}$ and $\Delta$ abs) to C) $\mathrm{NO}_{2}$ and $\mathrm{D}$ ) $\mathrm{NH}_{3}$ gases. 


\section{"Medium Density" SWNT Films}

A)

C)


B)



D)

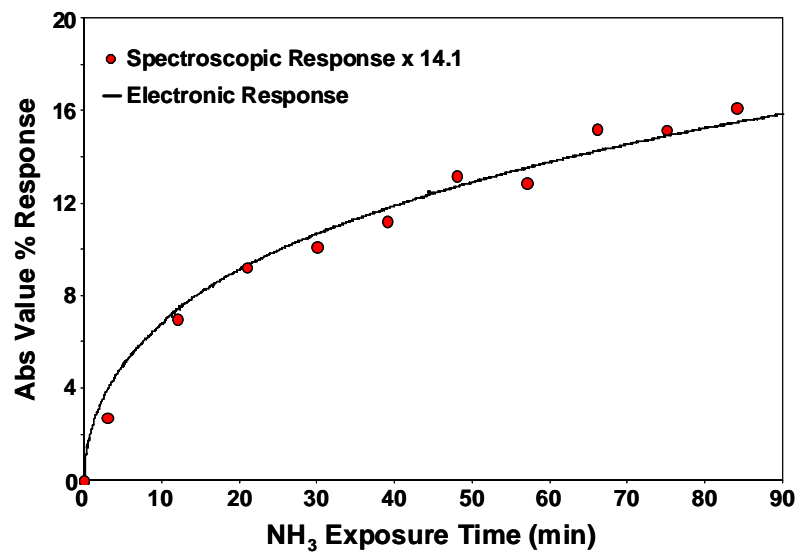

Figure S3. "Medium density" device output under A) $\mathrm{NO}_{2}$ and $\mathrm{B}$ ) $\mathrm{NH}_{3}$ gases. Absolute value of the device spectroscopic and electronic response (\% $\Delta \mathrm{G}$ and $\Delta \mathrm{abs}$ ) to C) $\mathrm{NO}_{2}$ and D) $\mathrm{NH}_{3}$ gases. 


\section{"Low Density" SWNT Films}

A)

C)
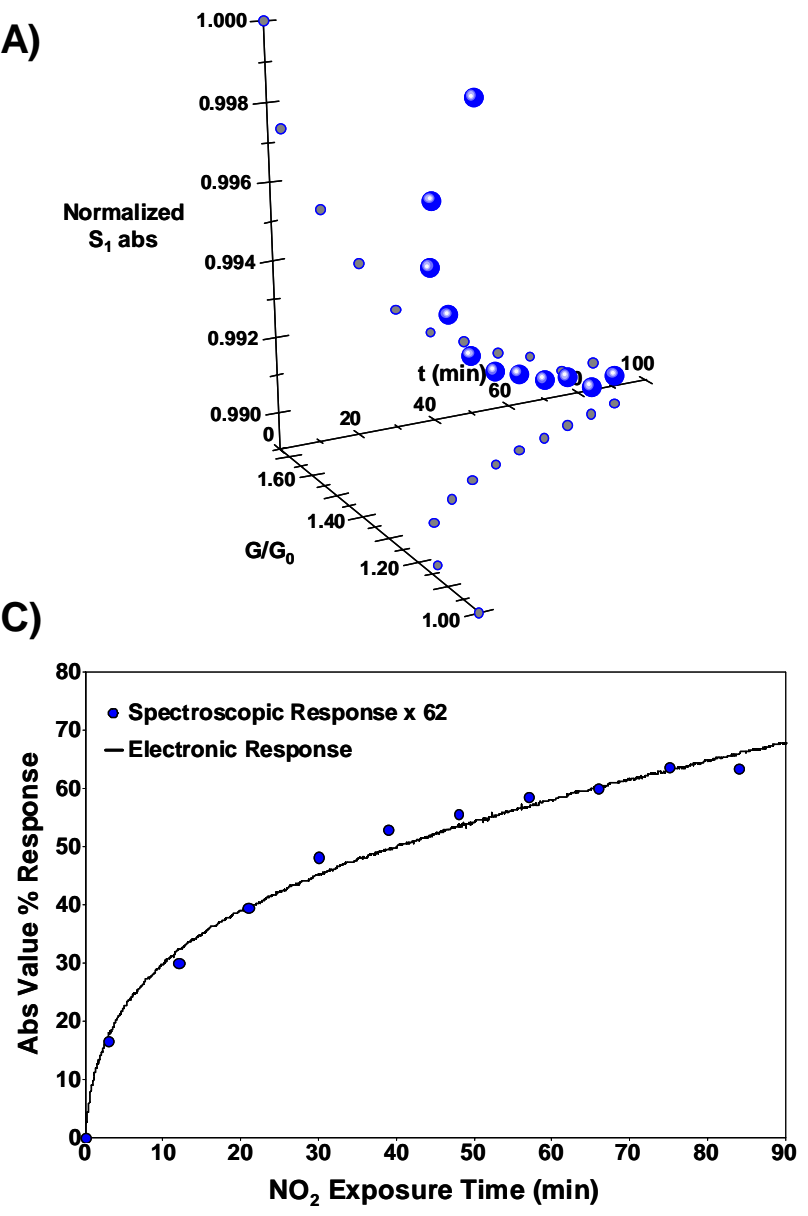

B)

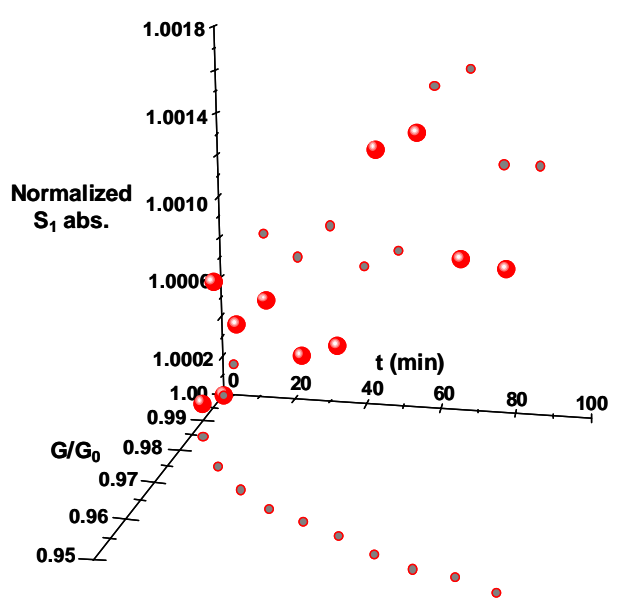

D)

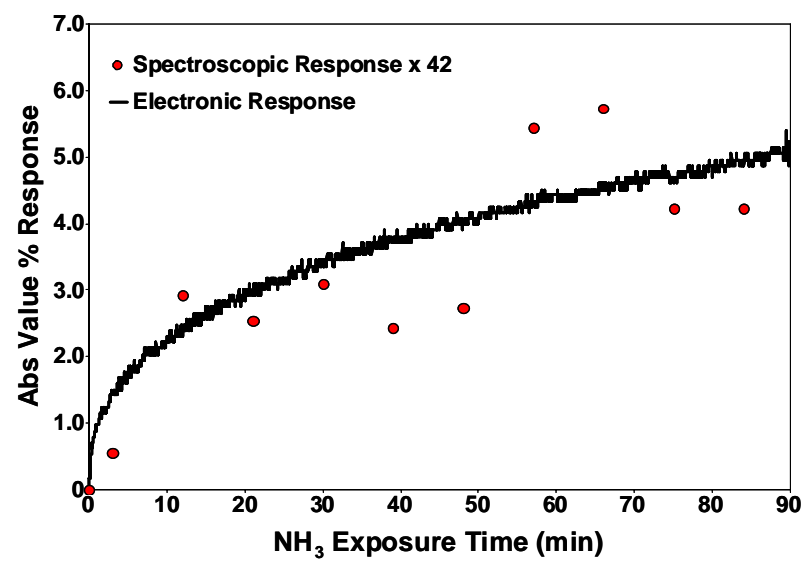

Figure S4. "Low density" device output under A) $\mathrm{NO}_{2}$ and B) $\mathrm{NH}_{3}$ gases. Absolute value of the device spectroscopic and electronic response (\% $\Delta \mathrm{G}$ and $\Delta$ abs) to C) $\mathrm{NO}_{2}$ and $\mathrm{D}$ ) $\mathrm{NH}_{3}$ gases. While $\mathrm{NO}_{2}$ exposure created significant electronic response $\mathrm{NH}_{3}$ exposure resulted in only a $\sim 5 \%$ change. Furthermore the spectroscopic response to $\mathrm{NH}_{3}$ was very small ( $\left.\sim 1 \%\right)$, this was just within the sensitivity limits of the spectrophotometer and resulted in the significantly noisier plot presented in part D. 